\title{
How to improve disease management of chronically ill patients? Perception of telemetric ECG recording and a novel software application
}

\author{
Michael Lang*, Stefan Ringbauer, Martin Mayr and Lukas Cepek \\ Nervenfachärztliche Gemeinschaftspraxis Ulm Pfauengasse 8, 89073 Ulm, Germany
}

\begin{abstract}
Background and Objective: During the last years, mobile applications and telemetry have been discussed to become an attractive tool to support and monitor patients with chronic diseases. This study aimed to evaluate the usability and perception of both telemetry and a novel app in MS patients scheduled for an ECG due to medication adjustment or follow-up.

Methods: In 211 neurological centers throughout Germany, telemetric 2-channel ECG was applied in 1082 patients with fingolimod prescriptions, who have to be subjected to ECG recording as a mandatory monitoring step. In a second approach, patients were offered to use the PatientConcept app, an application designed to ease patient-physician contact and to support patients in their daily life. Patients as well as physicians from the participating centers were surveyed to evaluate handling, acceptance and utility of both devices.
\end{abstract}

Results: The majority of patients perceived telemetric ECG recording as an interesting tool and appreciated shorter waiting times. $95 \%$, $95 \%$ and $98 \%$ of the centers believed that using telemetric ECG recording eliminates waiting times, accelerates medical reporting and provides a relief for test persons, respectively. Both, patients and physicians perceived the use of PatientConcept app as useful.

Conclusion: Being both well accepted, telemetric ECG recording and the use of a mobile app might provide new concepts to improve patient care. The positive results gained in this study may help to develop new perspectives for using these tools in the future, which may constitute a relief for the complex management of chronic diseases.

\section{Introduction}

Representing the most common cause of death and disability worldwide, chronic diseases are associated with a high need for patient care [1]. Management of most chronic diseases usually requires extensive patient education and long periods of supervision, including regular monitoring and routine visits to observe disease progress or complications [2]. These time- and cost-consuming demands place a considerable strain on patients, physicians and health service resources.

One example is the necessity of frequent electrocardiograms (ECG), representing one of the most important non-invasive tools for the diagnosis of cardiac diseases [3]. Patients treated with medications that potentially interfere with cardiac function have to be monitored regularly through an ECG. For instance, multiple sclerosis patients receiving fingolimod (Gilenya) have to be subjected to an ECG prior to and 6 hours after the first or adjusted dose of fingolimod. Telemetric monitoring systems offer the opportunity of recording ECGs outside of the cardiologist's practice and transmitting patients' physiologic parameters to a specialist for assessment. Telemetry ECG devices thereby allow easy and fast ECG monitoring of patients with suspected cardiac issues [3].

During the last years, also the use of mobile applications has gained importance and has been discussed to become an attractive tool to support and monitor patients $[4,5]$. Recently, the PatientConcept App was implemented into clinical routine. With the intention to support chronically ill patients in their daily life and to intensify the contact between patient and physician, the app facilitates monitoring and disease management [6]. Delivering healthcare via telemetry and mobile health (mHealth) devices might thus be a promising and costsaving tool to improve health outcomes and to relieve patients and physicians.

Obtaining physicians' and patients' perception constitutes an essential step in understanding the feasibility and long-term values when applying telemetric ECG or using PatientConcept App. Therefore, the FASTER study aimed to evaluate the acceptance of telemetric ECG measurement in MS patients scheduled for an ECG due to medication adjustment or follow-up. Furthermore, the study was initiated to address the perception of employing a software application in order to support patients in a comprehensive manner.

*Correspondence to: Michael Lang, Nervenfachärztliche Gemeinschaftspraxis Ulm Pfauengasse 8, 89073 Ulm, Germany, Tel: 00497316566 5; Fax 0049731 6542 0; E-mail: lang@neurologie-ulm.de

Key words: adherence, chronic disease, disease management, $m$ Health, monitoring, telemetry

Received: July 01, 2019; Accepted: July 09, 2019; Published: July 11, 2019 


\section{Methods}

\section{Study design}

The non-interventional FASTER (Focus on therapy associated monitoring and evaluation of telemedicine in real world setting) study was conducted between March 2016 and September 2017 in up to 300 practices and clinics in Germany. Multiple sclerosis patients with fingolimod prescriptions were included in the study. The primary goal was to investigate the feasibility and acceptance of telemetric 2-channel ECG measurements as a part of the mandatory monitoring requirements by both, patients and physicians. In a second approach, acceptance of an individualized mobile application was evaluated. Age and EDSS were also documented. The study was conducted in accordance with the 1964 Helsinki declaration and its later amendments or comparable ethical standards. The study design was approved by the local ethics committee of the Landesärztekammer Baden-Württemberg (F-2016013) Informed consent was obtained from all participants prior to study inclusion.

\section{Telemetric ECG}

During a visit at the neurologist / MS treatment center, telemetric ECGs (2-channel, four electrodes, 40 seconds) were performed on site using PhysioMem PM 100. External nurses (flying nurses) could be requested by the treatment center to support telemetric measures. ECG records were pseudonymized by applying time stamps (date and time of day) and immediately transmitted wirelessly to the analyzing center (ExCard Research GmbH) within a defined time frame (9 a.m. - 5 p.m.). Time stamps and patient data could only be allocated by the center performing the ECG. Experts / cardiologists of the analyzing center promptly analyzed the received ECG records. The medical report was sent back to the treating neurologist / treatment center by email within a maximum of 1 hour (Figure 1A). As the neurologist received the medical report from the experts / cardiologist, this monitoring setup required no additional personal appointment of the patient with the cardiologist.

\section{Evaluation of ECG functionality and acceptance}

To evaluate the functionality of the applied ECG, the total and evaluable number of ECGs was documented by the participating neurological centers. In addition, the necessity to repeat the transmission of ECG data, quality of data transfer and ECG records, processing time and occurring adverse events were assessed. Using a standardized printed questionnaire (yes/no questions), physicians and patients evaluated whether telemetric ECG was perceived as an interesting tool and a general relief, and whether it can reduce waiting hours and accelerate medical reporting.

\section{PatientConcept app}

The Patient Concept app has been recently implemented into clinical routine. It is available for free download via the German app store (for both iOS and android smartphones or tablets, usable in a multilingual manner) [6]. Patients included in the study were offered

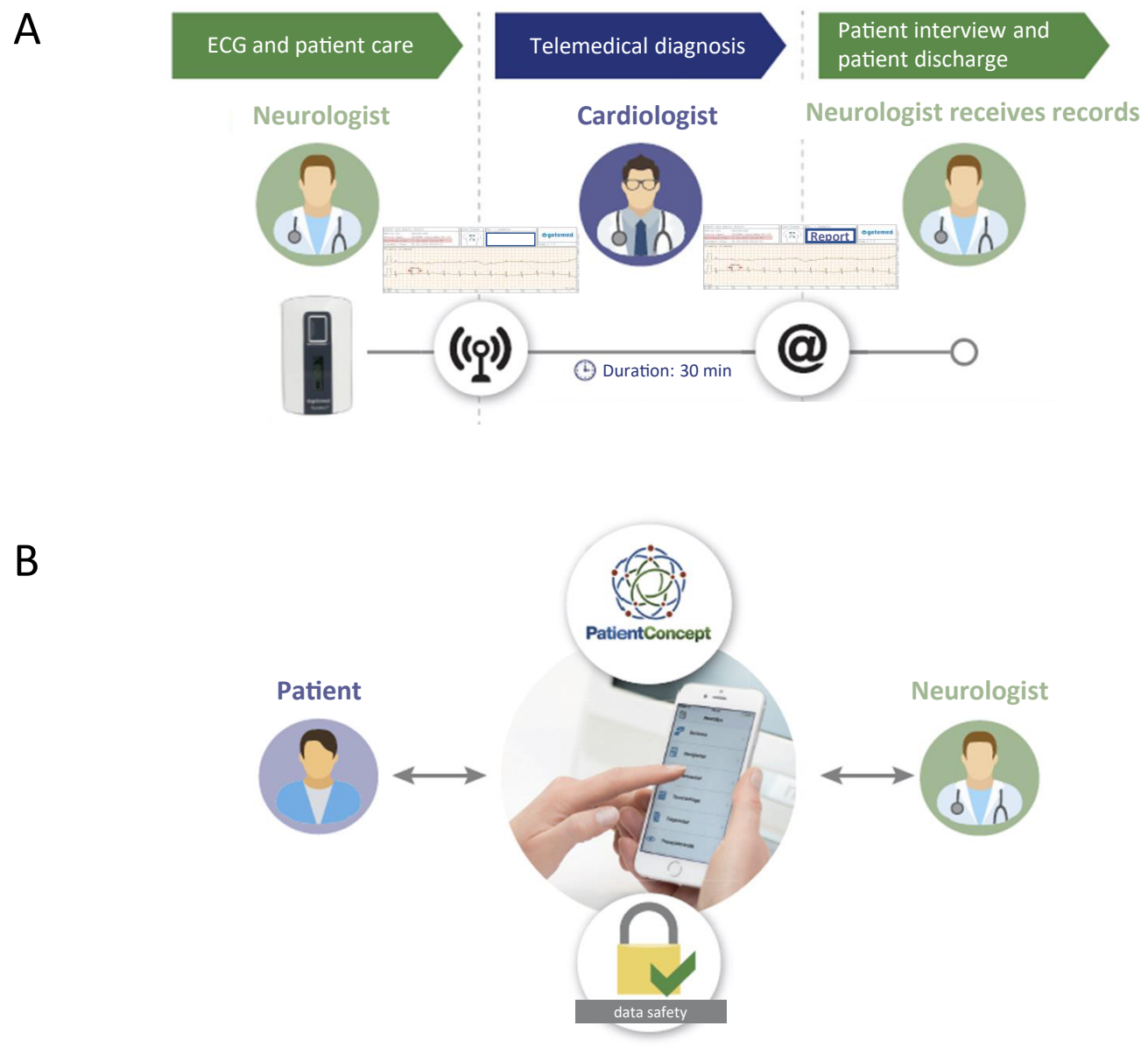

Figure 1. Study design 
to use the app during the last 6 months of the investigation period (due to developmental reasons the app was not available at an earlier timepoint). Patients were allowed to make use of all available modules and to employ the app at their own discretion. Webcast-trainings were offered to educate physicians and patients on the usage of the app and the corresponding backend.

\section{Evaluation of the acceptance of app usage}

Patients as well as physicians from the participating centers completed standardized questionnaires (yes/no questions) to evaluate the use of PatientConcept app. Patients assessed whether app usage was beneficial or useful to communicate with the center, whether it may reduce consultations, and whether patients were satisfied with the available app features. Physicians evaluated whether they perceived the app as useful to increase adherence and whether they showed interest in testing the application. Patients answered questionnaires via their smartphone, physicians via the backend (dashboard of the application).

\section{Statistics}

Data were analyzed descriptively using the program R. For categorial variables, percentages in relation to the total number were provided. For metric variables, mean values were provided.

\section{Results}

\section{Quality of telemetric ECG}

Telemetric ECG was applied to 1082 test persons with a median age of 39 years (range 16-71) and a median EDSS of 2 (range 0-8) in 211 participating centers throughout Germany. 1538 ECGs were recorded (in parts, patients received repeated recording, 5.1\% of records were not evaluable). On average, the medical report was available in the recording center after 12 minutes.

\section{Support by flying nurses}

During the study, 197 nurses were requested to support ECG measurements for a minimum of 1 to a maximum of 7 hours. 17\% of treating centers requested a nurse. The reasons for external request included limited capacities due to lacking staff or lacking willingness for education of staff $(50 \%)$. Overall, the use of external nurses was regarded as very positive (score 1.3 on a 6 -step scale from $1=$ very good to $6=$ insufficient). Only $1.8 \%$ reported delays or communication problems when requesting a nurse.

\section{Acceptance of telemetric ECG recording}

731 patients from 166 centers participated in the survey. The necessity of digital communication between patients and physicians was valued with a mean score of 2.5 (on a 6-step scale from 1 representing high need to 6 representing no need). $80 \%$ of participants stated that usage of telemedicine tools is beneficial. $73 \%$ and $19 \%$ of participants rated the offer of telemetric ECG recording in the treatment center as very good and good, respectively.

The vast majority of patients perceived telemetric ECG recording as an interesting tool and appreciated fast medical reporting and shorter waiting times for ECG measurements (Figures 2A, B and E). Patients also experienced a general relief due to telemetric ECG recording (Figure 2D). Rating levels regarding usability were independent of increasing age. A minimum of $93 \%$ of patients affirmed the use of telemetric ECG.

Patients also assessed whether telemetry may enrich communication, displaying a largely positive evaluation throughout the patient cohort (Figure $2 \mathrm{C}$ ). In contrast to telemetry usability, utility of telemetry for communication purposes was less well accepted with increasing age. While more than $80 \%$ of patients younger than 40 years recognized telemetric tools as enrichment for communication, older patients showed reduced acceptance.

In addition, 211 physicians from the participating centers evaluated the application of telemetric ECG recording with regard to usability, quality and benefit. $98 \%$ of treating centers classified this device as an interesting supporting tool (Figure 3). 95\%, 95\% and $98 \%$ of the centers believed that using telemetric ECG recording eliminates waiting times, accelerates medical reporting and provides a relief for test persons, respectively. In only $6 \%$ of cases waiting times or communication problems were reported, while technical procedures by the involved companies were assessed positive by $96 \%$ of the patients. The quality of data sets was considered high and was rated with a mean score of 2.5 (on a 6 -step scale from 1 representing excellent to 6 representing insufficient).

\section{Acceptance of app usage}

The perception of app usage was analyzed in a cohort of 92 patients (Figure $4 \mathrm{~A}$ ). $51 \%$ of users stated that the app was a useful tool to communicate with the treatment center (Figure $4 \mathrm{~A}$ ). $36 \%$ of users believed that the app might reduce the number of consultations. $92 \%$ of patients were satisfied with currently available features and did not propose any particular requests regarding further app functions. Most commonly used modules included 'medication (pill) alarm', 'appointment request' and 'information'. Analyses of the nature of the use by $92 \mathrm{MS}$ patients revealed that $64 \%$ of these patients used more than one and $30 \%$ four or more modules. For instance, 59\%, 56\% and $44 \%$ of patients using the module 'opening hours' also employed the modules 'appointment inquiry', 'follow-up prescription' and 'medication alarm', respectively. Patients using the module 'therapy control' also employed the modules 'news' (79\%), 'seminars' (71\%), 'medication alarm' (71\%), 'laboratory values' (57\%) and 'memory function' (50\%).

In general, elderly patients showed less interest in communications via app (Figure $2 \mathrm{~F}$ ), while $53.4 \%$ of patients aged younger than 40 years were interested in using an app to communicate with their treating physician.

Based on the experiences gained when using this software application, $50 \%$ of the centers believed that this novel application might represent a useful tool to increase adherence.

\section{Discussion}

Physicians' and patients' acceptance of an intervention or the use of a particular device is an essential prerequisite for its successful application. This study analyzed the acceptance of applying telemetric ECG for mandatory monitoring and of using a recently developed supportive app. Usage of telemetric ECG recording was well recognized by physicians as well as patients that have to be subjected to an ECG due to monitoring requirements. Participating patients and centers considered this approach as an overall relief and perceived telemetric ECG as useful and time-saving. This result is supported by studies reporting that telemetric follow-up of implantable electronic cardiac devices had a high degree of acceptance among both, patients as well as health care professionals, and carried the potential to reduce health care costs $[7,8]$. Also home-monitoring of implantable cardiac devices with telemetric functionalities is becoming increasingly popular because it allows remote diagnosis of proper device functionality and also optimization of the device settings. Thus, telemetric ECG measures can 
A

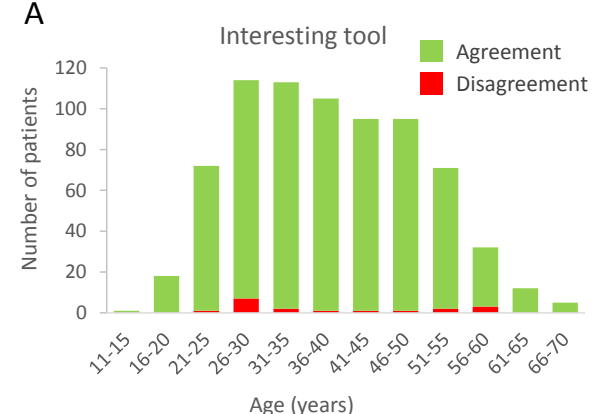

D

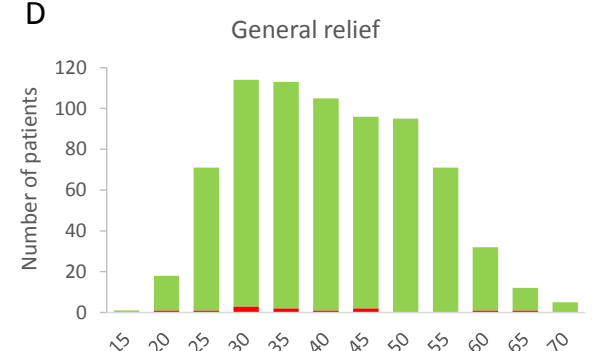

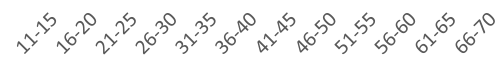

Age (years)
B

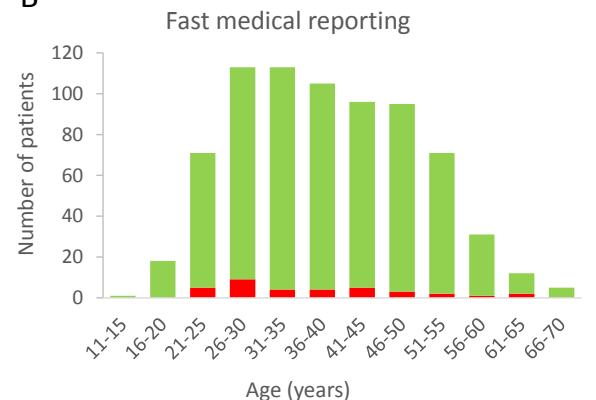

E

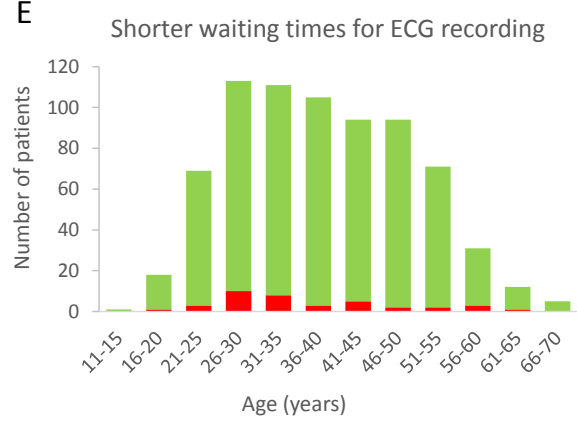

Age (years)
C

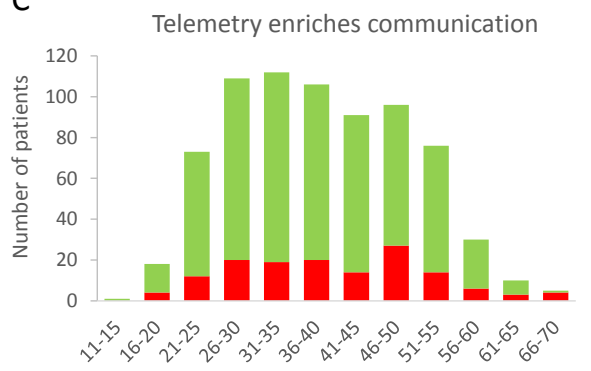

Age (years)

F

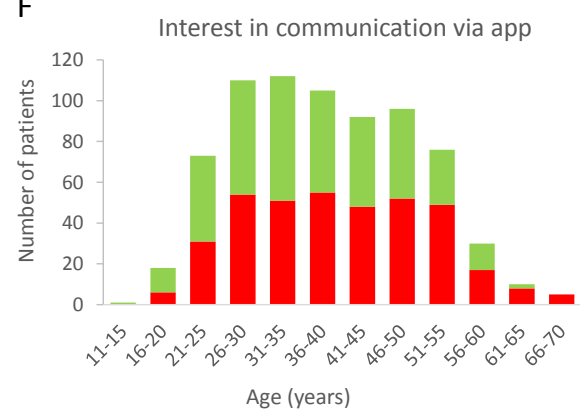

Figure 2. Patients' evaluation of telemetric ECG measurement

Evaluation of telemetric ECG $(n=211)$

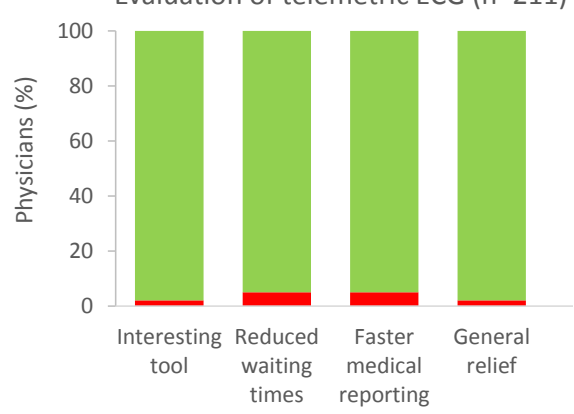

Agreement $\square$ Disagreement

Figure 3. Physicians` evaluation of telemetric ECG measurement

A

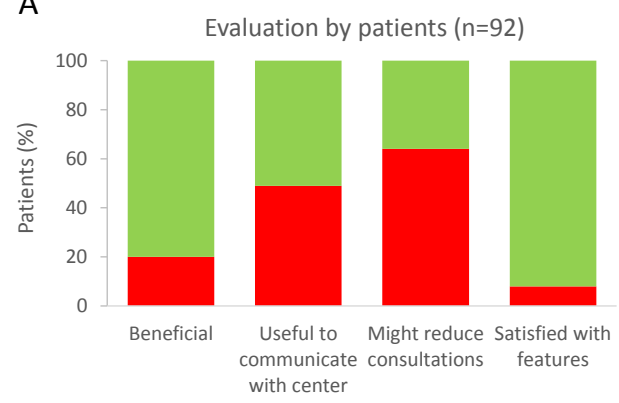

B

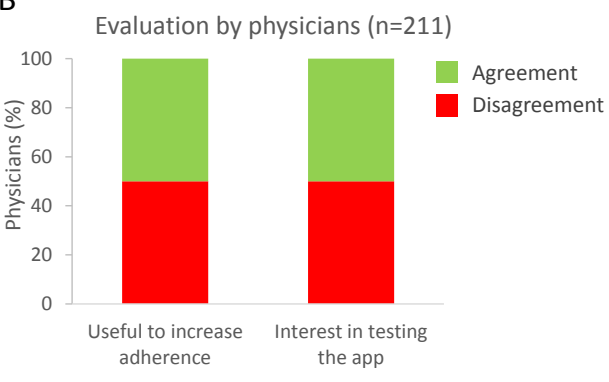

Figure 4. Evaluation of app usage 
be helpful for monitoring purposes and may facilitate implementation of complex therapy regimens, emphasizing the growing interest in digital health of physicians, patients, and technology companies [9].

ECG recording requires the clinician to be well trained in the technical use of the equipment [10]. In order to overcome a potential lack of adequately trained personnel in the MS centers, trained flying nurses were available to technically support the treating centers. Every fifth center requested support by a nurse, and the support was rated positively throughout.

Besides telemetric measurements, mobile health devices have gained importance in disease management [5]. Entitled as the new edge on healthcare innovation, mHealth potentially may deliver healthcare regardless of time and place, evading not only geographical and temporal, but also organizational barriers [11,12]. In this study, patients were offered to use and evaluate a novel and innovative software application [6]. Patients used the app in a multifunctional manner. Half of the patients testing the app felt that it is a useful tool to communicate with the treating center, whereby younger users were less skeptical than older patients. According to a survey by a German health insurance company, every second patient would appreciate the opportunity to contact or communicate with their physician online [13]. Enhanced communication may help to increase patients' commitment to the practice and thereby facilitate continuous support of chronically ill patients. These patients in particular, facing long-term medication are known to have low adherence rates [14]. As medication adherence is critical to achieving improved health outcomes, quality of life, and cost-effective health care [1], there is a high need for innovative approaches to improve adherence. In this context, intensified patient support via patient support programs (through patient academy, MS nurse or physicians) has already been shown to be beneficial for improving adherence in earlier studies [15]. Furthermore, a positive influence of mHealth-concepts on adherence [16,17] with high usability and acceptability for mobile interventions has been reported [18]. A similar tendency is reflected in the presented study with $50 \%$ of participating centers considering the application as a useful tool to increase adherence. Given the general annual increase in mobile phone users [19], the importance of mHealth is likely to increase in the future.

Limitations of the study include the small number of patients evaluating the use of PatientConcept App, which can be explained by the late availability of the application only in the last months of the investigation period. Therefore, further studies should investigate app acceptance, usability and its impact on adherence in a larger patient population covering a longer time period.

In conclusion, this study implies that new concepts of telemetric ECG recording and the use of a mobile app might improve patient management. Applied approaches were well recognized and perceived as useful in the practice by both, patients and physicians. The positive results gained in this study can help to develop new perspectives for future use of these tools, which may constitute a relief for patient monitoring and complex management of chronic diseases.

\section{Authorship}

M. Lang and L. Cepek substantially contributed to conception and design, acquisition and interpretation of data. S. Ringbauer substantially contributed to interpretation of data. M. Mayr substantially contributed to conception and design and analysis of data. All authors revised the article critically for important intellectual content, gave their final approval of the version to be published, and take public responsibility for appropriate portions of the content.

\section{Acknowledgments}

Editorial support (writing assistance, based on authors' detailed directions) was provided by Katrin Blumbach (med:unit $\mathrm{GmbH}$ ).

\section{Funding}

The study was supported by Novartis Pharma. Patient Concept system has received financial support from the Bavarian state, Neuropoint Patient Academy and Systemhaus Ulm, Bayer and Sanofi Genzyme.

\section{Competing interest}

M. Lang and L. Cepek have received travel grants, speaker's honoraria, financial research support, consultancy fees from Teva, Merck Serono, Genzyme -Sanofi, Novartis, Bayer, Biogen, Roche. S. Ringbauer and M. Mayr have nothing to disclose.

\section{References}

1. Viswanathan M, Golin CE, Jones CD (2012) Interventions to improve adherence to self-administered medications for chronic diseases in the United States: a systematic review. Ann Intern Med 157: 785-795.

2. Reynolds R, Dennis S, Hasan I (2018) A systematic review of chronic disease management interventions in primary care. BMC Fam Pract 19: 11

3. Marouf M, Vukomanovic G, Saranovac L, Bozic M (2017) Multi-purpose ECG telemetry system. Biomed Eng Online 16: 80. [Crossref]

4. Elwyn G, Hardisty AR, Peirce SC, May C, Evans R, et al. (2012) Detecting deterioration in patients with chronic disease using telemonitoring: navigating the 'trough of disillusionment'. J Eval Clin Pract 18: 896-903. [Crossref]

5. Organization WH. mHealth. New horizons for health through mobile technologies.

6. Lang M, Mayr M, Ringbauer S, Cepek L (2019) PatientConcept App: Key Characteristics, Implementation, and its Potential Benefit. Neurol Ther 8: 147-154.

7. Bosch R, Mutscher I (2017) Telemetric follow-up of implantable electronic cardiac devices: Optimisation of care in clinical practice. Herzschrittmacherther Elektrophysiol 28: 260-267.

8. Hilbel T, Helms TM, Mikus G, Katus HA, Zugck C (2008) Telemetry in the clinical setting. Herzschrittmacherther Elektrophysiol 19: 146-154.

9. Birnbaum F1, Lewis D2, Rosen RK3, Ranney ML4 (2015) Patient engagement and the design of digital health. Acad Emerg Med 22: 754-756. [Crossref]

10. Lefkowitz RJ (1975) Identification of adenylate cyclase-coupled beta-adrenergic receptors with radiolabeled beta-adrenergic antagonists. Biochem Pharmacol 24: 16511658. [Crossref]

11. Hood ML (2018) The role of technology and telemetry medicine in the initial management of a ST-segment elevated myocardial infarction in a rural emergency department. Rural Remote Health 18: 4478.

12. Akter S, Ray P (2010) mHealth - an Ultimate Platform to Serve the Unserved. Yearb Med Inform. [Crossref]

13. Tachakra S, Wang XH, Istepanian RS, Song YH (2003) Mobile e-health: the unwired evolution of telemedicine. Telemed J E Health 9: 247-257. [Crossref]

14. Krankenkasse TT. Studie im Auftrag der Techniker Krankenkasse zeigt: Apps bahnen sich den Weg in die Gesundheitsversorgung.

15. Burkhart PV, Sabaté E (2003) Adherence to long-term therapies: evidence for action. $J$ Nurs Scholarsh 35: 207. [Crossref]

16. Hamine S, Gerth-Guyette E, Faulx D, Green BB, Ginsburg AS (2015) Impact of mHealth chronic disease management on treatment adherence and patient outcomes: a systematic review. J Med Internet Res 17: e52. [Crossref]

17. Yasmin F, Banu B, Zakir SM, Sauerborn R, Ali L, et al. (2016) Positive influence of short message service and voice call interventions on adherence and health outcomes in case of chronic disease care: a systematic review. BMC Med Inform Decis Mak 16: 46. [Crossref] 
Lang M (2019) How to improve disease management of chronically ill patients? Perception of telemetric ECG recording and a novel software application

18. Anglada-Martinez H, Riu-Viladoms G, Martin-Conde M, Rovira-Illamola M, SotocaMomblona JM, et al. (2015) Does mHealth increase adherence to medication? Results of a systematic review. Int J Clin Pract 69: 9-32.
19. Payne HE, Lister C, West JH, Bernhardt JM (2015) Behavioral functionality of mobile apps in health interventions: a systematic review of the literature. JMIR Mhealth Uhealth 3: e20.

20. Statista (2018) Number of mobile phone users worldwide from 2015 to 2020.

Copyright: (O2019 Lang M. This is an open-access article distributed under the terms of the Creative Commons Attribution License, which permits unrestricted use, distribution, and reproduction in any medium, provided the original author and source are credited. 